\title{
Letters to the
}

\section{Editor}

\section{CorCap device in addition to mitral surgery in heart failure: Is it truly beneficial? \\ To the Editor:}

The recent report about mitral valve surgery in heart failure ${ }^{1}$ raises a number of interesting questions. While left ventricular (LV) end-diastolic volume and end-systolic volume in the treatment group decreased significantly more with the cardiac support device (CorCap; Acorn Cardiovascular, St Paul, Minn) than in the control group, other differences were statistically insignificant. Important end points such as survival, ejection fraction, and measurable indices of functional capacity (6-minute walking test and oxygen consumption), although presented as "better" in the treatment group, were not statistically different. The only adverse event that was nearing significance $(P=.06)$ in favor of the control group, namely a neurologic deficit/stroke, is hidden in Table 2. The lack of significant advantages to the treatment group in the presence of a similarly stable mitral repair is intriguing. The CorCap device is enveloping both ventricles, defining a new upper limit to total (end-diastolic) biventricular volume. We are all familiar with the echocardiographic picture of the spherical shape of the globally enlarged LV, including the interventricular septum. Obviously, by enveloping both ventricles from the outside, the device does not restrict or reshape the septal component of LV dilatation. Moreover, assuming that the tension of the device is even and equal throughout, this means that we are expecting to limit LV free-wall dilatation by suspending it on the right ventricle (RV). Can we trust the RV with its much lower pressures and wall tension to successfully counteract and restrict either the systolic or the diastolic distention of the globally or even the regionally enlarged LV? It is likely that the greater reduction in LV volumes observed in the treatment group is the result of a new balance between the two ventricles. RV volume is restricted more than LV volume. The RV being dragged or "squeezed" to- ward the LV during each systole, and having decreased space for diastolic filling because of the rightward bulging, noncompliant ventricular septum, can now produce a smaller stroke volume. LV end-diastolic volume is consequently decreased, with some improvement in shape, but probably with minimal change in wall stress and ejection fraction beyond what has already been accomplished with mitral repair or replacement. The authors conclude that in addition to the clear benefit from elimination of mitral regurgitation, there is significant additional benefit with the CorCap device.

The question remains whether the mechanical preload reduction of the LV via $\mathrm{RV}$ restriction, which does not improve $\mathrm{LV}$ contractile function, outweighs the adverse events and the risks and technical difficulties encountered when such a patient requires heart transplantation.

Amir Elami, $M D$ Department of Cardiothoracic Surgery Hadassah University Hospital Jerusalem, Israel

\section{Reference}

1. Acker MA, Bolling S, Shemin R, Kirklin J, Oh JK, Mann DL, et al. Mitral valve surgery in heart failure: insights from the Acorn Clinical Trial. J Thorac Cardiovasc Surg. 2006; 132:568-77.

doi:10.1016/j.jtcvs.2006.09.058

\section{Microbiologically documented nosocomial infections after coronary artery bypass surgery without cardiopulmonary bypass \\ To the Editor:}

In the latest issue of the Journal of Thoracic and Cardiovascular Surgery, we read with great interest the article by Falagas and colleagues, ${ }^{1}$ in which they evaluated the frequency, characteristics, and predisposing factors of microbiologically documented nosocomial infections in a welldefined subgroup of critically ill patients undergoing off-pump coronary artery bypass grafting. In this clearly and well-documented 